\title{
Globe
}

Revue internationale d'études québécoises

\section{Denise Deshaies et Diane Vincent [éd.] : Discours et constructions identitaires, Québec, Presses de l'Université Laval, 2004}

\section{Katri Suhonen}

Volume 10, numéro 1, 2007

URI : https://id.erudit.org/iderudit/1000119ar

DOI : https://doi.org/10.7202/1000119ar

Aller au sommaire du numéro

Éditeur(s)

Globe, Revue internationale d'études québécoises

ISSN

1481-5869 (imprimé)

1923-8231 (numérique)

Découvrir la revue

Citer ce compte rendu

Suhonen, K. (2007). Compte rendu de [Denise Deshaies et Diane Vincent [éd.] : Discours et constructions identitaires, Québec, Presses de l'Université Laval,

2004]. Globe, 10(1), 180-186. https://doi.org/10.7202/1000119ar d'utilisation que vous pouvez consulter en ligne.

https://apropos.erudit.org/fr/usagers/politique-dutilisation/ 
relations France-Bas-Canada avant 1855 et à bien décrire l'importance et surtout les limites de l'événement lui-même, les organisateurs du colloque et les participants ont fortement contribué à la compréhension de ce que le président québécois de la Commission franco-québécoise sur les lieux communs de mémoire, Marcel Masse, a appelé de ses vœux, soit une approche qui tienne compte des aspects historiques, ethnologiques, sociologiques, scientifiques et artistiques ${ }^{22}$. Tout en regrettant et en comprenant la portion congrue réservée à l'aval de l'événement, sans doute beaucoup trop riche pour faire l'objet d'autre chose que de coups de sonde bien choisis, il faut ici rendre hommage aux éditeurs et aux organisateurs du colloque, pour ce livre magnifique qui nous entraîne dans un voyage riche et passionnant aux sources d'une identité toujours en construction.

Sylvain Simard

Député de Richelieu

Président de la Commission de la culture

Assemblée nationale du Québec

\section{Denise Deshaies et Diane Vincent [éd.] \\ Discours et constructions identitaires \\ Québec, Presses de l'Université Laval, 2004.}

Composé autour d'une réflexion entamée lors du séminaire "Discours et constructions identitaires" (la discipline, le niveau et les activités du séminaire menant à la rédaction du collectif ne sont pas expliqués), l'ouvrage dirigé par Denise Deshaies et Diane Vincent, regroupant des contributions de chercheurs chevronnés et d'étudiants, a pour objectif de circonscrire diverses facettes de la construction identitaire par le biais de discours (surtout sur un "nous français" au Canada (p. XI)) :

Si la langue constitue pour plusieurs communautés un symbole qui permet aux individus de se définir et de définir leur rapport aux autres, elle est également l'outil par lequel se construit l'identité [...]. Les collaborateurs à cet ouvrage souscrivent à une telle position, abordant la question identitaire à travers les marques linguistiques qui façonnent les représentations. (p. X; je souligne)

$$
+\div+
$$

22. Marcel MASSE, "Mot de bienvenue», p. VIII. 
Le corpus étudié va des discours politiques (le général de Gaulle, la ministre Diane Lemieux) aux discours journalistiques (articles d'un magazine acadien), administratifs (documents d'organismes autochtones) et historiques (ouvrages et réflexions d'un historien, récits de voyage), en passant par des corpus oraux composés d'interviews et d'observations auprès de divers groupes minoritaires et par le «discours" pictural véhiculé par le panthéon philatélique canadien.

Compte tenu de l'étendu du corpus, la notion de discours est chargée dans ce recueil de multiples sens et l'approche privilégiée par les analyses reste parfois éloignée des visées premières. S'il est intéressant de lire la réflexion des auteurs sur l'identité des communautés abordées, il est moins sûr que les corpus textuels étudiés témoignent de sa construction. Plusieurs études font état des identités collectives ou individuelles telles que celles-ci sont exprimées ou décrites - et non construites - par les discours tenus. De plus, les "marques linguistiques qui façonnent les représentations" identitaires dans les textes sont peu exploitées. En effet, le discours et l'analyse $d u$ discours (l'approche revendiquée par plusieurs chapitres du livre) étant des notions non équivoques dont la signification differe d'une discipline à l'autre, voire à l'intérieur d'une même discipline ${ }^{23}$, il aurait été pertinent de préciser le sens réservé à ces notions dans le contexte de ce recueil. En revanche, le deuxième thème-clé de l'ouvrage, l'identité, est circonscrit avec clarté par Louis-Jacques Dorais. L'auteur rappelle quelques éléments de base (l'identité est à la fois individuelle et collective et sa construction est dépendante de l'interaction avec d'autres membres d'une communauté donnée) et s'attarde ensuite à la définition de trois types d'identité collective: culturelle (''altérité ou la similarité d'un individu ou d'un groupe par rapport aux autres), ethnique (la conscience d'une différence généalogique, linguistique, socio-économique ou autre entre plusieurs groupes à l'intérieur d'un État) et nationale (la conscience d'appartenir à un peuple qui occupe un territoire précis). Ces définitions reposent sur des évaluations subjectives, elles sont liées à des rapports de pouvoir au profit des uns et aux dépens des autres et elles ne sont pas des catégories isolées (par exemple, identité nationale et ethnique entrent en conflit dans une société multiculturelle).

$$
+4
$$

23. Un aperçu sur la diversité définitoire des termes mentionnés est disponible dans Dominique MaINGUENEAU, Les cermes cles de l'analyse du discours, Paris, Seuil, coll. «Mémo ”, 1996, p. 11-12, 28-29. 
La contribution la plus fidèle à la méthodologie de l'analyse du discours est l'étude de Jean-Michel Adam (l'un des fondateurs de la discipline) sur l'art rhétorique dans le discours prononcé par Charles de Gaulle à Montréal le 24 juillet 1967. Adam rappelle le soutien inconditionnel du général au nationalisme francophone en Amérique du Nord et décortique les diverses composantes linguistiques du discours qui renforcent ce message. Les faits de langue exposés permettent d'établir une confiance entre orateur et public, d'effriter la frontière entre individu, homme d'État, État et nation, d'accentuer le dit par le non-dit, de privilégier le logos, l'ethos ou le pathos à diverses parties du discours, de structurer l'argumentation selon la logique raisonnement-justification-confirmation, de juxtaposer des termes issus de champs sémantiques divers ou d'amplifier le message par l'hyperbole. Cette analyse de la matière textuelle du discours analyse qui expose les phénomènes implicites au service du message explicite - offre un excellent exemple de l'analyse linguistique comme complément de l'analyse historique ou sociologique. Qui plus est, Adam illustre, avec des exemples fort intéressants, l'effacement de certains traits mentionnés de la version disponible sur Internet, procédure qui neutralise la force idéologique de l'original (enregistré et archivé).

Après cette excellente ouverture, le lecteur est en quelque sorte laissé sur sa faim, car plusieurs études subséquentes exposent de longs passages de textes écrits ou oraux sans pour autant en analyser la matérialité textuelle, les composantes discursives proprement dites. Cet effet aurait pu être évité en précisant que certains chapitres abordent les discours dans le dessein d'en faire sortir des éléments linguistiques (et implicites), alors que d'autres se limitent à la discussion du contenu thématique (et explicite) des textes. Les analyses auraient également gagné à être regroupées selon le type de discours étudiés (documents administratifs, historiques, journalistiques ou interviews), les types d'identités abordées (identité linguistique doublée d'une identité nationale, ethnique ou sexuelle) ou la discipline qui encadre l'analyse (linguistique, histoire, sociologie). Une subdivision aurait permis d'aborder le discours dans une perspective différente d'un sous-chapitre à l'autre et peut-être d'en approfondir l'analyse par des parallèles, comparaisons et conclusions.

Nonobstant le changement dans le programme de lecture qui suit l'analyse d'Adam, les études offrent des réflexions intéressantes sur les identités qui se manifestent dans les textes et les discours produits par les membres de diverses collectivités. Louis-Pascal Rousseau étudie la définition de l'identité métisse (terme polysémique motivé par des facteurs 
généalogiques, historiques, culturels, juridiques ou géographiques; dans le contexte canadien, la descendance francophone est un facteur déterminant) dans deux types de documents écrits, produits par deux communautés dans les Territoires du Nord-Ouest (le formulaire d'adhésion à la communauté et le "texte constitutionnel" qui justifie la qualification identitaire). Il s'attarde à la définition et à la proportion des qualificatifs qui caractérisent les membres dans les documents et confirme, sans surprise, que chaque document multiplie les termes faisant référence à la communauté respective. Karine Gauvin, pour sa part, étudie un corpus d'articles journalistiques, tirés du magazine acadien Ven'd'est entre 1989 et 1995, afin d'exposer deux visions contraires sur l'identité acadienne. D'une part, s'y dessine une identité "de cœur" et "sans frontières" qui mise sur un sentiment d'appartenance motivé par des liens généalogiques et historiques. D'autre part, y prend forme le manifeste d'une identité active et politique par la population qui vit sur les lieux de l'Acadie historique, qui assume concrètement son existence minoritaire et qui œuvre quotidiennement pour ses droits et pour la survie de la communauté (l'observation de quelques traits de langue soutient l'analyse, telle l'utilisation du déterminant défini pluriel pour désigner l'identité collective des Acadiens de l'Atlantique, contrairement au déterminant défini singulier pour souligner la dispersion et la précarité des Acadiens de la diaspora). Monica Heller complète la discussion sur les francophonies au Canada par l'analyse de l'évolution de l'identité au șein d'une communauté franco-ontarienne, telle qu'elle se manifeste dans les échanges lors des réunions d'un centre communautaire. Heller expose d'abord le contexte historique et socio-économique de la communauté et du centre pour ensuite illustrer un progrès idéologique, visible dans les prises de parole des membres, qui finit par modifier le fonctionnement de l'organisme: à partir d'une idéologie valorisant la nation canadiennefrançaise et concentrée sur la réclamation des ressources et des droits, l'organisme évolue vers la valorisation d'une identité francophone ouverte à quiconque utilise la langue pour divers besoins et met l'accent sur les services offerts à la communauté.

Sur la base des entrevues réalisées auprès des professionnels de l'enseignement au Québec (le choix de l'échantillon aurait-il pu être justifié, par exemple, par le fait que ce groupe occupe une position idéale pour transmettre son opinion?), Wim Remysen affirme que, si la langue française reste une composante centrale de l'identité québécoise, la justification de cette importance repose sur des croyances stéréotypées. Les discours officiels (politique et artistique) étudiés par Remysen confirment ce résultat et 
l'auteur en conclut que les stérétypies ont un rôle de premier ordre dans la formation d'un sentiment d'identité (il encourage d'ailleurs avec raison l'analyse de l'utilité ou de la nocivité des stéréotypes dans la production identitaire aux dépens de l'étude de leur fondement). L'auteur se sert du modèle proposé par Monica Heller et Gabriele Budach pour interpréter les attitudes manifestées par les interviewés: le discours sur le rapport entre langue et identité des francophones au Canada peut être du type traditionaliste (valorisant la nation canadienne-française unie par l'héritage linguistique et religieux), modernisant (valorisant l'État-nation québécois unilingue) ou mondialisant (valorisant le bilinguisme et la langue en tant que bien économique). Quand les interlocuteurs sont interrogés sur la place du français dans le patrimoine québécois, les réponses révèlent des attitudes des types traditionaliste et modernisant. Quant à la menace posée par l'anglais, les attitudes sont du type modernisant ou mondialisant (certains considèrent l'anglais comme un outil de travail - au même titre qu'un ordinateur - sans sentir leur identité francophone et québécoise menacée). L'auteur constate pourtant que le discours officiel n'encourage pas l'identité linguistique du type mondialisant. Les stéréotypes sont en jeu également dans la brève réflexion de Fouzia Benzakour qui a remarqué, en écoutant les jeunes immigrés maghrébins au Québec (sans préciser davantage le corpus, les méthodes ou l'hypothèse de l'étude), leur difficulté à se positionner par rapport à l'accent québécois: la présumée supériorité linguistique qu'ils se donnent en raison de leur français de référence est remplacée par la surprise de constater leur infériorité sociale dans le contexte québécois (difficultés à trouver du travail, obligation de refaire des études). La part réservée à l'analyse du discours dans cette contribution est marginale (mis à part les commentaires sur quelques phrases présentées hors contexte).

L'étude de Normand Labrie confronte deux visions sur la survie des minorités linguistiques. La vision traditionnelle considère que les minorités assurent leur pérennité par la reproduction biologique, la vie sédentaire, le caractère fermé de la communauté ainsi que par l'homogénéité de leur culture et population. Labrie conteste cette vision par la présentation des observations sur des groupes qui souffrent d'une double marginalité: les gais et lesbiennes francophones au Canada sont en position minoritaire par rapport à leur communauté linguistique en raison de leur orientation sexuelle et par rapport à leur communauté sexuelle en raison de leur langue maternelle. Il affirme que la condition de base pour la survie de leur identité linguistique et sexuelle est leur mobilité sociale et géographique. Il illustre ses propos par les entrevues réalisées auprès de six personnes homosexuelles de 
langue française qui exposent dans leur discours les raisons de leur survie en tant que minorité sexuelle (ascension sociale et déplacement géographique) et les traces de leur identité francophone (par exemple la langue vernaculaire).

Pour terminer, le collectif propose trois lectures de discours historiques. Mathieu Roy découvre une contradiction dans les discours ou articles produits par le nationaliste canadien Pierre Breton, au milieu du $\mathrm{XX} \mathrm{X}^{e}$ siècle et ses ouvrages publiés plus récemment. Les premiers manifestent une volonté de réfuter plusieurs fausses impressions sur le Québec dans le Canada anglophone ayant pour objectif d'empêcher la division du pays, d'illustrer la différence socioculturelle entre le Canada et les États-Unis différence due en grande partie au fait français - ainsi que d'encourager une vision progressiste du Canada. Par contre, les ouvrages publiés plus récemment témoignent d'une diminution de la place allouée au Québec. Malgré cette évolution (l'auteur propose quelques explications), les discours tenus par Breton participent à la construction d'une identité nationale canadienne distincte de l'identité étasunienne. Jean-François Dumouchel, pour sa part, étudie la description que font les missionnaires jésuites des peuples autochtones au XVII ${ }^{\mathrm{e}}$ siècle et en extrait quelques exemples qui exposent les qualités appréciées (richesse matérielle, puissance militaire et ouverture spirituelle) et appréhendées (violence, pauvreté, faiblesse) par les Européens. Le vocabulaire axiologique utilisé dans ce discours transforme la description en jugement et permet d'établir une hiérarchie entre les nations. S'il est intéressant de lire les témoignages de ces rencontres, la part réservée à la construction identitaire par le biais du discours reste à montrer, à moins de considérer la description des peuples comme contribution à la formation de leur identité (l'identité serait alors une donnée imposée de l'extérieur). Enfin, bien que l'article de Vincent Fontaine expose un phénomène certes intéressant - sur les 2064 timbres émis depuis 1851 au Canada, 324 honorent une personnalité canadienne dont 104 sont dédiés à une personnalité francophone -, sa part analytique se résume à un décompte et à une catégorisation des timbres sélectionnés (on présume que la présentation historique est motivée par les textes qui accompagnent les timbres, les sources étant laissées sans mention). Il aurait été bénéfique d'étayer l'apport de ce type de discours à la construction identitaire (outre la mention que le choix des sujets des timbres révèle des valeurs qui rassemblent les Canadiens).

Si la notion de l'identité (nationale, ethnique, culturelle, linguistique, sexuelle) est au cœur de chaque érude, la part réservée à l'analyse "des marques linguistiques" et des "faits de langue" dans les discours choisis demeure restreinte. Ce qui importe, c'est ce que disent les discours étudiés, aux dépens de comment ils le disent. Quelques précisions 
terminologiques auraient été les bienvenues ainsi qu'une division du matériel, afin de mieux servir la particularité de chaque discours, approche ou discipline et une conclusion, pour résumer la contribution des analyses à la compréhension des liens entre discours et identité. Nonobstant ces quelques réserves, je tiens à saluer les analyses menées, surtout par les étudiants, pour l'intérêt de leurs sujets ainsi que pour la qualité de l'écriture, de la documentation, de la structure et de l'argumentation.

Katri Suhonen

Université Concordia

\section{Jean Crête [éd.]}

Politiques publiques: le Québec comparé

Québec, Presses de l'Université Laval, 2006.

Ce livre, issu d'un colloque qui a eu lieu à l'Université Laval en 2002, déclare l'intention de parler du Québec, "d'un Québec mis en contexte, d'un Québec comparé." Je ne peux que saluer cette volonté de comprendre le Québec, vu ici sous l'angle de ses choix de politiques publiques, dans le contexte de l'expérience d'autres sociétés. Il y a en effet tout un corpus universitaire sur l'exceptionnalisme québécois, par exemple sur un modèle socio-économique nommé Québec Inc. ou modèle québécois, qui serait unique en Amérique du Nord. On entend souvent dans les médias et sur les tribunes politiques que le Québec aurait une culture plus socialdémocrate et plus portée à la concertation que celle de ses voisins. Il y a sans doute du bien-fondé dans ces affirmations, mais il nous manque toujours les études comparatives pour ajouter les nuances nécessaires et démontrer les limites de cet exceptionnalisme. Mais malgré la nécessité de poursuivre une démarche comparative, ce collectif n'augmente pas notre compréhension du Québec de manière importante. En effet, elle montre tous les défauts typiques d'un recueil de textes tirés d'un colloque : il y a plusieurs contributions intéressantes, mais la qualité et la pertinence des textes sont inégales, et la somme est moindre que les parties.

L'introduction essaie de situer la problématique du collectif autour de l'importance de la méthode comparative, quoique l'espace accordé à cette question (environ 6 pages) soit nettement insuffisant, laissant de côté plusieurs questions épistémologiques et méthodologiques courantes en ce qui a trait à la recherche sur les méthodes dans les domaines de la politique 